ANNALES

POLONICI MATHEMATICI

$84.3(2004)$

\title{
On the Dirichlet problem in the Cegrell classes
}

\author{
by Rafae Czyż (Kraków) and Per Ånag (Taipei)
}

\begin{abstract}
Let $\mu$ be a non-negative measure with finite mass given by $\varphi\left(d d^{c} \psi\right)^{n}$, where $\psi$ is a bounded plurisubharmonic function with zero boundary values and $\varphi \in$ $L^{q}\left(\left(d d^{c} \psi\right)^{n}\right), \varphi \geq 0,1 \leq q \leq \infty$. The Dirichlet problem for the complex Monge-Ampère operator with the measure $\mu$ is studied.
\end{abstract}

1. Introduction. Let $\Omega \subseteq \mathbb{C}^{n}, n \geq 2$, be a bounded hyperconvex domain, i.e., a bounded, connected and open set that admits a negative plurisubharmonic exhaustion function. A bounded plurisubharmonic function $u$ defined on $\Omega$ belongs to the class $\mathcal{E}_{0}$ if

$$
\lim _{\substack{z \rightarrow \xi \\ z \in \Omega}} u(z)=0
$$

for every $\xi \in \partial \Omega$, and

$$
\int_{\Omega}\left(d d^{c} u\right)^{n}<\infty
$$

where $\left(d d^{c} \cdot\right)^{n}$ is the complex Monge-Ampère operator. Let the measure $\mu$ defined on $\Omega$ be given by

$$
\mu=\varphi\left(d d^{c} \psi\right)^{n},
$$

where $\varphi \in L^{q}\left(\left(d d^{c} \psi\right)^{n}\right), \varphi \geq 0,1 \leq q \leq \infty$ and $\psi \in \mathcal{E}_{0}$. It is proved in Theorem 5.11 of [4] that every non-negative measure $\widetilde{\mu}$ defined on $\Omega$ can be decomposed into

$$
\widetilde{\mu}=\widetilde{\varphi}\left(d d^{c} \widetilde{\psi}\right)^{n}+\nu,
$$

where $\widetilde{\psi} \in \mathcal{E}_{0}$ and $\widetilde{\varphi} \in L_{\text {loc }}^{1}\left(\left(d d^{c} \widetilde{\psi}\right)^{n}\right), \widetilde{\varphi} \geq 0$. The non-negative measure $\nu$ is such that there exists a pluripolar set $A \subseteq \Omega$ such that $\nu(A)=\nu(\Omega)$. For $q=1$, the measure $\mu$ has finite mass and it puts no mass on pluripolar sets, by (1.1). Lemma 5.14 in [4] implies that there exists a unique function $u \in \mathcal{F}$

2000 Mathematics Subject Classification: Primary 32W20; Secondary 32U15.

Key words and phrases: complex Monge-Ampère operator, Dirichlet problem, plurisubharmonic function.

The research of the first-named author was partially supported by KBN, under grant number 1 P03A 03726. 
such that $\left(d d^{c} u\right)^{n}=\mu$ as measures defined on $\Omega$ (see Definition 2.1 for the class $\mathcal{F})$.

In this article some results concerning the solution of this Dirichlet problem will be obtained for the case $q>1$. If $q>1$ is such that $n(q-1) \geq 1$, then our Theorem 2.3 states that the unique solution $u \in \mathcal{F}$ belongs to $\mathcal{F}_{p}$, where $p=n(q-1)$ (see Definition 2.1 for the class $\mathcal{F}_{p}$ ). On the other hand, if $q>1$ is such that $n(q-1)<1$, then it is proved in Theorem 2.4 that the unique solution $u \in \mathcal{F}$ is such that

$$
\int_{\Omega}(-u)^{p}\left(d d^{c} u\right)^{n}<\infty
$$

where $p=n(q-1)$. Moreover there exists a decreasing sequence $\left[u_{j}\right]_{j=1}^{\infty}$, $u_{j} \in \mathcal{E}_{0}$, which converges pointwise to $u$ on $\Omega$ as $j$ tends to $\infty$ and satisfies

$$
\sup _{j} \int_{\Omega}\left(-u_{j}\right)^{p}\left(d d^{c} u_{j}\right)^{n}<\infty, \quad \sup _{j} \int_{\Omega}\left(d d^{c} u_{j}\right)^{n}<\infty .
$$

If there can be no misinterpretation a sequence $[\cdot]_{j=1}^{\infty}$ will be denoted by $[\cdot]$. The results of Theorems 2.3 and 2.4 will be extended to the corresponding classes with continuous boundary values. The note ends by recalling a theorem for the case when $q=\infty$ (see Theorem 2.7). For an introduction to pluripotential theory we recommend [7].

The authors would like to thank Jonas Andersson and Sławomir Kołodziej. They have both made many valuable comments on and suggestions for this manuscript.

2. Dirichlet problem. A domain is an open and connected set. A domain $\Omega \subseteq \mathbb{C}^{n}$ is called hyperconvex if there exists a plurisubharmonic function $\varphi: \Omega \rightarrow(-\infty, 0)$ such that the closure of the set

$$
\{z \in \Omega: \varphi(z)<c\}
$$

is compact in $\Omega$ for every $c \in(-\infty, 0)$. Throughout this note $\Omega$ will be a bounded hyperconvex domain in $\mathbb{C}^{n}, n \geq 2$.

DEFinition 2.1. Define $\mathcal{F}(=\mathcal{F}(\Omega))$ to be the class of plurisubharmonic functions $\varphi$ defined on $\Omega$ such that there exists a decreasing sequence $\left[\varphi_{j}\right]$, $\varphi_{j} \in \mathcal{E}_{0}$, which converges pointwise to $\varphi$ on $\Omega$ as $j$ tends to $\infty$, and

$$
\sup _{j} \int_{\Omega}\left(d d^{c} \varphi_{j}\right)^{n}<\infty .
$$

Let $p \geq 1$. If $\left[\varphi_{j}\right]$ can also be chosen such that

$$
\sup _{j} \int_{\Omega}\left(-\varphi_{j}\right)^{p}\left(d d^{c} \varphi_{j}\right)^{n}<\infty,
$$

then $\varphi$ is said to be in the class $\mathcal{F}_{p}\left(=\mathcal{F}_{p}(\Omega)\right)$. 
The classes $\mathcal{F}_{p}$ were first introduced in [3] and $\mathcal{F}$ in [4]. These classes are two of the so-called Cegrell classes. For further information about the Cegrell classes see, e.g., [3]-[6] and [9].

Lemma 2.2. If $\phi, \psi \in \mathcal{E}_{0}$, then for each $p \geq 0$,

$$
\int_{\Omega}(-\phi)^{p+n}\left(d d^{c} \psi\right)^{n} \leq C \int_{\Omega}(-\phi)^{p}\left(d d^{c} \phi\right)^{n},
$$

where $C \geq 0$ is a constant, depending only on $n, p$ and the supremum of $\psi$.

Proof. Cf. [1] (see also [2]).

Theorem 2.3. Let $\psi \in \mathcal{E}_{0}$ and $\varphi \in L^{q}\left(\left(d d^{c} \psi\right)^{n}\right), \varphi \geq 0, n \geq 2$. If $1<q<\infty$ is such that $n(q-1) \geq 1$, then there exists a unique function $u \in \mathcal{F}_{p}$ such that

$$
\left(d d^{c} u\right)^{n}=\varphi\left(d d^{c} \psi\right)^{n}
$$

where $p=n(q-1)$.

Proof. Let $\phi \in \mathcal{E}_{0}$. Hölder's inequality implies that

$$
\begin{aligned}
\int_{\Omega}(-\phi)^{p} \varphi\left(d d^{c} \psi\right)^{n} & \leq\left(\int_{\Omega} \varphi^{q}\left(d d^{c} \psi\right)^{n}\right)^{1 / q}\left(\int_{\Omega}(-\phi)^{p q /(q-1)}\left(d d^{c} \psi\right)^{n}\right)^{(q-1) / q} \\
& =C_{1}\left(\int_{\Omega}(-\phi)^{p+n}\left(d d^{c} \psi\right)^{n}\right)^{p /(n+p)},
\end{aligned}
$$

where $C_{1} \geq 0$ is a constant and $p=n(q-1)$. Since $p \geq 1$ by assumption, it follows from Lemma 2.2 that

$$
\int_{\Omega}(-\phi)^{p+n}\left(d d^{c} \psi\right)^{n} \leq C_{2} \int_{\Omega}(-\phi)^{p}\left(d d^{c} \phi\right)^{n},
$$

where $C_{2} \geq 0$ is a constant. Inequalities (2.1) and (2.2) imply that there exists a constant $A$ such that

$$
\int_{\Omega}(-\phi)^{p} \varphi\left(d d^{c} \psi\right)^{n} \leq A\left(\int_{\Omega}(-\phi)^{p}\left(d d^{c} \phi\right)^{n}\right)^{p /(p+n)}
$$

for every $\phi \in \mathcal{E}_{0}$, hence Theorem 5.1 in [3] shows that there exists a unique $u \in \mathcal{F}_{p}$ such that

$$
\left(d d^{c} u\right)^{n}=\varphi\left(d d^{c} \psi\right)^{n} .
$$

TheOREM 2.4. Let $\psi \in \mathcal{E}_{0}$ and $\varphi \in L^{q}\left(\left(d d^{c} \psi\right)^{n}\right), \varphi \geq 0, n \geq 2$. If $1<q<\infty$ is such that $n(q-1)<1$, then there exists a unique function $u \in \mathcal{F}$ such that $\left(d d^{c} u\right)^{n}=\varphi\left(d d^{c} \psi\right)^{n}$ and

$$
\int_{\Omega}(-u)^{p}\left(d d^{c} u\right)^{n}<\infty
$$


where $p=n(q-1)$. Moreover there exists a decreasing sequence $\left[u_{j}\right], u_{j} \in \mathcal{E}_{0}$, which converges pointwise to $u$ on $\Omega$ as $j$ tends to $\infty$ and satisfies

$$
\sup _{j} \int_{\Omega}\left(-u_{j}\right)^{p}\left(d d^{c} u_{j}\right)^{n}<\infty, \quad \sup _{j} \int_{\Omega}\left(d d^{c} u_{j}\right)^{n}<\infty .
$$

Proof. Let $\phi \in \mathcal{E}_{0}$. By using the same technique as in the proof of Theorem 2.3 it follows that there exists a constant $A$ not depending on $\phi$ such that

$$
\int_{\Omega}(-\phi)^{p} \varphi\left(d d^{c} \psi\right)^{n} \leq A\left(\int_{\Omega}(-\phi)^{p}\left(d d^{c} \phi\right)^{n}\right)^{p /(n+p)} .
$$

In particular, this shows that the measure $\varphi\left(d d^{c} \psi\right)^{n}$ vanishes on pluripolar sets. Lemma 5.14 in [4] implies that there exists a unique $u \in \mathcal{F}$ such that

$$
\left(d d^{c} u\right)^{n}=\varphi\left(d d^{c} \psi\right)^{n},
$$

since the given measure has finite total mass. Let $u_{j} \in \mathcal{E}_{0}$ be such that

$$
\left(d d^{c} u_{j}\right)^{n}=\min (j, \varphi)\left(d d^{c} \psi\right)^{n} .
$$

The comparison principle shows that the sequence $\left[u_{j}\right]$ is decreasing and converges pointwise to $u$ on $\Omega$ as $j$ tends to $\infty$. Inequality (2.3) implies that

$$
\begin{aligned}
\int_{\Omega}\left(-u_{j}\right)^{p}\left(d d^{c} u_{j}\right)^{n} & =\int_{\Omega}\left(-u_{j}\right)^{p} \min (j, \varphi)\left(d d^{c} \psi\right)^{n} \\
& \leq \int_{\Omega}\left(-u_{j}\right)^{p}\left(d d^{c} u\right)^{n} \leq A\left(\int_{\Omega}\left(-u_{j}\right)^{p}\left(d d^{c} u_{j}\right)^{n}\right)^{p /(n+p)} .
\end{aligned}
$$

This yields

$$
\int_{\Omega}\left(-u_{j}\right)^{p}\left(d d^{c} u_{j}\right)^{n} \leq A^{(p+n) / n}
$$

hence

$$
\sup _{j} \int_{\Omega}\left(-u_{j}\right)^{p}\left(d d^{c} u_{j}\right)^{n} \leq A^{(p+n) / n} .
$$

Inequality (2.3) implies that

$$
\int_{\Omega}\left(-u_{j}\right)^{p}\left(d d^{c} u\right)^{n} \leq A\left(\int_{\Omega}\left(-u_{j}\right)^{p}\left(d d^{c} u_{j}\right)^{n}\right)^{p /(n+p)} \leq A^{(p+n) / n} .
$$

By the monotone convergence theorem,

$$
\lim _{j \rightarrow \infty} \int_{\Omega}\left(-u_{j}\right)^{p}\left(d d^{c} u\right)^{n}=\int_{\Omega}(-u)^{p}\left(d d^{c} u\right)^{n} .
$$

Combining (2.4) and (2.5) yields

$$
\int_{\Omega}(-u)^{p}\left(d d^{c} u\right)^{n} \leq A^{(p+n) / n} .
$$


The next step is to generalize the results in Theorems 2.3 and 2.4 to some more general classes of bounded plurisubharmonic functions. Recall that the Perron-Bremermann envelope is defined by

$$
\operatorname{PB}_{f}(z):=\sup \left\{w(z): w \in \mathcal{P} \mathcal{S H}(\Omega), \limsup _{\substack{\zeta \rightarrow \xi \\ \zeta \in \Omega}} w(\zeta) \leq f(\xi) \forall \xi \in \partial \Omega\right\},
$$

where $\mathcal{P S H}(\Omega)$ denotes the class of plurisubharmonic functions defined on $\Omega$ and $f: \partial \Omega \rightarrow \mathbb{R}$ is a given function. Recall that if $\Omega$ is a bounded hyperconvex domain and $f: \partial \Omega \rightarrow \mathbb{R}$ is a continuous function, then $\mathrm{PB}_{f} \in$ $\mathcal{P S H}(\Omega)$.

DEFinition 2.5. Let $\mathcal{K} \in\left\{\mathcal{E}_{0}, \mathcal{F}_{p}, \mathcal{F}\right\}$ and $f: \partial \Omega \rightarrow \mathbb{R}$ be a continuous function such that

$$
\lim _{\substack{z \rightarrow \xi \\ z \in \Omega}} \operatorname{PB}_{f}(z)=f(\xi)
$$

for every $\xi \in \partial \Omega$. A plurisubharmonic function $u$ defined on $\Omega$ belongs to the class $\mathcal{K}(f)(=\mathcal{K}(\Omega, f))$ if there exists a function $\varphi \in \mathcal{K}$ such that

$$
\mathrm{PB}_{f} \geq u \geq \varphi+\mathrm{PB}_{f} .
$$

REMARK. If $\mathcal{K} \in\left\{\mathcal{E}_{0}, \mathcal{F}_{p}, \mathcal{F}\right\}$, then $\mathcal{K}(0)=\mathcal{K}$.

REMARK. Theorem 2.3 is also valid for the Cegrell class $\mathcal{F}_{p}(f)$.

The classes $\mathcal{E}_{0}(f)$ and $\mathcal{F}_{p}(f)$ were first introduced in [3] and the class $\mathcal{F}(f)$ in [9].

THEOREM 2.6. Assume that $f: \partial \Omega \rightarrow \mathbb{R}$ is a continuous function such that

$$
\lim _{\substack{z \rightarrow \xi \\ z \in \Omega}} \operatorname{PB}_{f}(z)=f(\xi)
$$

for every $\xi \in \partial \Omega$, and $\mathrm{PB}_{f}+\mathrm{PB}_{-f} \in \mathcal{E}_{0}$. Let $\psi \in \mathcal{E}_{0}$ and $\varphi \in L^{q}\left(\left(d d^{c} \psi\right)^{n}\right)$, $\varphi \geq 0, n \geq 2$. If $1<q<\infty$ is such that $n(q-1)<1$, then there exists a unique function $u \in \mathcal{F}(f)$ such that $\left(d d^{c} u\right)^{n}=\varphi\left(d d^{c} \psi\right)^{n}$ and

$$
\int_{\Omega}\left(-u-\mathrm{PB}_{-f}\right)^{p}\left(d d^{c} u\right)^{n}<\infty
$$

where $p=n(q-1)$. Moreover there exists a decreasing sequence $\left[u_{j}\right], u_{j} \in$ $\mathcal{E}_{0}(f)$, which converges pointwise to $u$ on $\Omega$ as $j$ tends to $\infty$ and satisfies

$$
\sup _{j} \int_{\Omega}\left(-u_{j}-\mathrm{PB}_{-f}\right)^{p}\left(d d^{c} u_{j}\right)^{n}<\infty, \quad \sup _{j} \int_{\Omega}\left(d d^{c} u_{j}\right)^{n}<\infty .
$$

Proof. From Theorem 2.4 it follows that there exists $v \in \mathcal{F}$ such that

$$
\left(d d^{c} v\right)^{n}=\varphi\left(d d^{c} \psi\right)^{n}, \quad \int_{\Omega}(-v)^{p}\left(d d^{c} v\right)^{n}<\infty
$$


where $p=n(q-1)$. Theorem 7.4 in [9] implies that there exists $u \in \mathcal{F}(f)$ such that $\left(d d^{c} u\right)^{n}=\varphi\left(d d^{c} \psi\right)^{n}$. The function $v+\mathrm{PB}_{f}$ belongs to $\mathcal{F}(f)$ and

$$
\left(d d^{c} u\right)^{n}=\left(d d^{c} v\right)^{n} \leq\left(d d^{c}\left(v+\mathrm{PB}_{f}\right)\right)^{n},
$$

hence

$$
v+\mathrm{PB}_{f} \leq u,
$$

by Corollary 7.7 in [9]. Thus

$$
\int_{\Omega}\left(-u-\mathrm{PB}_{-f}\right)^{p}\left(d d^{c} u\right)^{n} \leq \int_{\Omega}\left(-v-\mathrm{PB}_{f}-\mathrm{PB}_{-f}\right)^{p}\left(d d^{c} v\right)^{n}<\infty .
$$

Now for the second part of the theorem. Theorem 4.10 in [9] together with the assumption that $\mathrm{PB}_{f}+\mathrm{PB}_{-f} \in \mathcal{E}_{0}$ implies that there exists $u_{j} \in \mathcal{E}_{0}(f)$ such that

$$
\left(d d^{c} u_{j}\right)^{n}=\min (j, \varphi)\left(d d^{c} \psi\right)^{n} .
$$

Moreover $\left[u_{j}\right]$ is a decreasing sequence which converges pointwise to $u$ on $\Omega$ as $j \rightarrow \infty$, and

$$
\sup _{j} \int_{\Omega}\left(d d^{c} u_{j}\right)^{n}<\infty .
$$

Furthermore, $u_{j}+\mathrm{PB}_{-f} \in \mathcal{E}_{0}$. The assertion then follows by repeating the argument in the proof of Theorem 2.4.

Theorem 2.7. Let $\psi \in \mathcal{E}_{0}$ and $\varphi \in L^{q}\left(\left(d d^{c} \psi\right)^{n}\right), \varphi \geq 0, n \geq 2$. If $q=\infty$, then there exists a unique $u \in \mathcal{E}_{0}(f)$ such that $\left(d d^{c} u\right)^{n}=\varphi\left(d d^{c} \psi\right)^{n}$. In particular, if $f=0$, then there exists a unique $u \in \mathcal{E}_{0}$ such that $\left(d d^{c} u\right)^{n}=$ $\varphi\left(d d^{c} \psi\right)^{n}$.

Proof. There exists a constant $c \geq 0$ such that

$$
\varphi\left(d d^{c} \psi\right)^{n} \leq c\left(d d^{c} \psi\right)^{n}=\left(d d^{c}\left(c^{1 / n} \psi\right)\right)^{n},
$$

hence there exists a unique $u \in \mathcal{E}_{0}(f)$ such that $\left(d d^{c} u\right)^{n}=\varphi\left(d d^{c} \psi\right)^{n}$, by the proof of Theorem 7.4 in [9].

REMARK. Theorem 2.7 is a special case of Theorem A in [8].

\section{References}

[1] Z. Błocki, Estimates for the complex Monge-Ampère operator, Bull. Polish Acad. Sci. Math. 41 (1993), 151-157.

[2] - , The Complex Monge-Ampère Operator in Pluripotential Theory, lecture notes, manuscript, 2002.

[3] U. Cegrell, Pluricomplex energy, Acta Math. 180 (1998), 187-217.

[4] - The general definition of the complex Monge-Ampère operator, Ann. Inst. Fourier (Grenoble) 54 (2004), 159-179.

[5] R. Czyż, Convergence in capacity of the Perron-Bremermann envelope, IMUJ Preprint 2004/3, Jagiellonian Univ. 
[6] R. Czyż and P. Åhag, On the Cegrell classes, IMUJ Preprint 2004/11, Jagiellonian Univ.

[7] M. Klimek, Pluripotential Theory, Oxford Sci. Publ., Oxford Univ. Press, 1991.

[8] S. Kołodziej, The range of the complex Monge-Ampère operator, II, Indiana Univ. Math. J. 44 (1995), 765-782.

[9] P. Åhag, The complex Monge-Ampère operator on bounded hyperconvex domains, Ph.D. thesis, Umeå Univ., 2002.

Institute of Mathematics

Jagiellonian University

Reymonta 4

30-059 Kraków, Poland

E-mail: Rafal.Czyz@im.uj.edu.pl
Institute of Mathematics

Academia Sinica

Taipei 11529, Taiwan

E-mail: per@math.sinica.edu.tw

Reçu par la Rédaction le 25.11.2004 